\title{
Cerclaje profiláctico en mujeres con nacimientos prematuros espontáneos previos, asociados con infección bacteriana ascendente
}

\author{
Alfredo Ovalle S. ${ }^{1}$, Oscar Valderrama C. ${ }^{1}$, Gustavo Rencoret P. ${ }^{1}$, Ariel Fuentes G. 1 , \\ María José del Río V. 1, Elena Kakarieka W. 2, María Angélica Martínez T. 3, Dagoberto \\ Pizarro S. $^{1}$ \\ 1 Servicio y Departamento de Obstetricia, Ginecología y Neonatología, Hospital San Borja Arriarán. Facultad de Medici- \\ na, Universidad de Chile. ${ }^{2}$ Servicio de Anatomía Patológica, Hospital San Borja Arriarán. 3 Programa de Microbiología, \\ I.C.B.M. Facultad de Medicina, Universidad de Chile.
}

\section{RESUMEN}

Objetivo: Determinar la eficacia del cerclaje cervical profiláctico en pacientes con embarazos únicos, cérvix $>25 \mathrm{~mm}$ e historia de nacimientos prematuros espontáneos, asociados con infección bacteriana ascendente (IBA). Métodos: Estudio clínico en pacientes con embarazos únicos y partos prematuros y/o abortos de $2^{\circ}$ trimestre espontáneos previos, sin partos de término, asociados con IBA. Se incluyeron los casos con longitud cervical de $\geq 25 \mathrm{~mm}$ al ingreso. Se comparó el cerclaje cervical hecho en pacientes derivadas antes de las 20 semanas, con el manejo expectante en mujeres enviadas después de esta edad gestacional y que mantuvieron longitud cervical $>25 \mathrm{~mm}$ durante los controles. Se excluyeron embarazadas con cérvix $<25 \mathrm{~mm}$ al ingreso del estudio, con acortamiento cervical $<25 \mathrm{~mm}$ durante el manejo con conducta expectante y mujeres con nacimientos prematuros previos sin histología placentaria. Resultados: Se incluyeron 51 pacientes, 23 con cerclaje y 28 sin cerclaje. El cerclaje cervical profiláctico, redujo significativamente la frecuencia del nacimiento prematuro $<37$ semanas $(4,3 \%$ vs $35,7 \%),<34$ semanas $(4,3 \%$ vs $28,6 \%)$ y la corioamnionitis histológica $(4,3 \%$ vs $32,1 \%)$, OR (IC95\%) $0,08(0,09-0,70), 0,11(0,01-0,99)$ y $0,01(0,01-$ $0,83)$, respectivamente. Conclusiones: En pacientes con nacimientos prematuros previos asociados con IBA, embarazo único y longitud cervical $>25 \mathrm{~mm}$, el cerclaje profiláctico, reduce la frecuencia del nacimiento prematuro $<37$ y $<34$ semanas y de la corioamnionitis histológica.

\section{PALABRAS CLAVE: Cerclaje, parto prematuro, infección bacteriana ascendente, corioamnionitis histológica}

\section{SUMMARY}

Aims: Determine the effectiveness of prophylactic cerclage in women with singleton pregnancies, cervix $>25$ $\mathrm{mm}$ and a history of spontaneous premature deliveries associated to ascending bacterial infection (ABI). Methods: Women with singleton pregnancies and history of preterm births, with no full-term deliveries, associated to $A B I$. Cases with $\geq 25 \mathrm{~mm}$ cervical length at admission were included. Cervical cerclage performed on patients derived before 20 weeks of pregnancy was compared to the expectant management of women submitted at a later gestational stage with sustained cervical length of $>25 \mathrm{~mm}$. Pregnant women with $<25$ $\mathrm{mm}$ cervix at referral, with cervical shortening $<25 \mathrm{~mm}$ at expectant management, and women with previous 
preterm birth without placental histology were excluded. Results: 51 patients were included, 23 with cerclage and 28 without cerclage. Prophylactic cerclage significantly reduced the frequency of premature birth $<37$ weeks, $4.3 \%$ vs $35.7 \%$ and $<34$ weeks, $4.3 \%$ vs $28.6 \%$ and histologic chorioamnionitis $4.3 \%$ vs $32.1 \%$ (9/28), OR (95\%Cl) $0.08(0.09-0.70), 0.11(0.01-0.99)$ and 0.01 (0.01-0.83), respectively. Conclusions: In patients with preterm births associated to $A B I$, singleton pregnancy and cervical length $>25 \mathrm{~mm}$, prophylactic cerclage reduces the frequency of premature delivery $<37$ and $<34$ weeks as well as histologic chorioamnionitis.

\section{KEY WORDS: Cerclage, preterm birth, ascending bacterial infection, histologic chorioamnionitis}

\section{INTRODUCCIÓN}

El parto prematuro es la principal causa de morbilidad y mortalidad perinatal en todo el mundo. Es responsable del 75 a $80 \%$ de la mortalidad perinatal, de más de la cuarta parte de la morbilidad a largo plazo y de la mayoría de las complicaciones neurológicas, respiratorias y gastrointestinales de los neonatos (1).

La infección bacteriana ascendente (IBA) según algunos autores es muy frecuente entre las 20 y 32 semanas de gestación. Está presente como factor de riesgo de nacimiento espontáneo, en $90 \%$ a las 24 semanas y $60 \%$ a las 32 semanas (2). Representó el $33 \%$ de las muertes fetales entre 22 y 30 semanas principalmente por neumonía (3) y se presentó como factor de riesgo en el $51 \%$ de los partos prematuros espontáneos entre 22 y 34 semanas en el Hospital Clínico San Borja Arriarán (4). Por otro lado está descrito que mientras menor es la longitud cervical, el riesgo de infección e inflamación intraamniótica es mayor (5-7).

Son diversas las intervenciones médicas realizadas durante el embarazo para prevenir el parto prematuro, por infección ascendente, y para reducir la morbilidad y la mortalidad perinatal asociada $(8,9)$. Pesquisa y tratamiento en población de riesgo de infecciones cérvicovaginales (vaginosis bacteriana, Streptococcus agalactiae) y urinarias; detección de fibronectina fetal y pesquisa rutinaria del acortamiento cervical en el segundo trimestre (9). Sin embargo, las tasas de prematurez no se han reducido con estas medidas.

El cerclaje no ha mostrado beneficios en mujeres con sólo nacimientos prematuros (10), sólo la longitud cervical acortada $(11,12)$ u otros factores de riesgo para parto prematuro. Los primeros estudios randomizados que evaluaron la eficacia del cerclaje indicado por ultrasonido mostraron resultados contradictorios (13-15). Un meta-análisis reciente (16), demostró que en embarazadas con partos prematuros previos y longitud cervical $<25 \mathrm{~mm}$, el cerclaje previno el nacimiento prematuro y redujo la mortalidad perinatal y la morbilidad compuesta. No existe información relacionada con la indicación de cerclaje en embarazos únicos con partos prematuros previos asociados con IBA y cérvix $>25 \mathrm{~mm}$.

El objetivo de este trabajo fue determinar la eficacia del cerclaje cervical profiláctico en pacientes con embarazos únicos, cérvix $>25 \mathrm{~mm}$ e historia de nacimientos prematuros espontáneos asociados con IBA.

\section{PACIENTES Y MÉTODOS}

Población estudiada. Estudio clínico, sobre la eficacia del cerclaje profiláctico en pacientes con embarazos únicos, sin partos de término e historia de nacimientos espontáneos asociados con IBA, atendidos en el Servicio de Obstetricia y Ginecología del Hospital San Borja Arriarán, en el período entre el 1 de enero de 2007 hasta el 31 de diciembre de 2010. El Comité de Ética aprobó la realización de este estudio. Las pacientes consintieron informadamente ingresar al estudio.

Se definió nacimiento prematuro espontáneo previo asociado con IBA, el aborto de $2^{\circ}$ trimestre (entre 15 y 21,6 semanas) y/o parto prematuro (entre 22 y 35,0 semanas) con los siguientes hallazgos anatomopatológicos específicos de infección ascendente: corioamnionitis aguda, funisitis aguda (2,3,17-19), o síndrome de infección del saco amniótico (SISA): neumonía fetal congénita con muerte fetal, asociada a corioamnionitis histológica (3), y con una o varias de las siguientes condiciones clínicas, sugerentes de infección intrauterina: rotura prematura de membranas (RPM), corioamnionitis clínica, sangrado vaginal con desprendimiento amniocorial, infección cérvicovaginal (ICV) por Streptococcus Grupo B (SGB), infección del tracto urinario (ITU), membranas prolapsadas bajo el orificio cervical externo (MPr), desprendimiento placentario, sepsis congénita $(7,8,17-23)$.

La edad gestacional se determinó por historia menstrual segura o por biometría fetal ultrasonográfica efectuada antes de las 20 semanas. 
Criterios de inclusión. Se recomendó cerclaje cervical a las mujeres que se derivaron antes de las 20 semanas al Policlínico de Alto Riesgo del hospital y con longitud cervical $\geq 25 \mathrm{~mm}$ (grupo estudio). No se hizo cerclaje, a las pacientes derivadas desde las 20 semanas, que mantuvieron longitud cervical $>25 \mathrm{~mm}$ durante el embarazo (grupo control), criterio imperante en el Servicio, las que se manejaron con conducta expectante.

Criterios de exclusión. Embarazo múltiple, mujeres con nacimientos prematuros previos sin estudio histológico placentario, embarazadas con cérvix $<25 \mathrm{~mm}$ en la admisión y con acortamiento cervical $<25 \mathrm{~mm}$ durante la conducta expectante.

El cerclaje lo realizó un solo operador (OV) antes de las 20 semanas, con técnica de MacDonald y con sutura Ethibon no 1, después de diagnosticar y tratar las infecciones genitourinarias. Todas las embarazadas se controlaron hasta el parto con ultrasonografía mensual que incluyó medición del cérvix.

No se usó progesterona en cualquiera de sus formas de presentación en ninguna de las pacientes.

Estudios microbiológicos. Se hizo pesquisa de la ICV (vaginitis aeróbica, vaginosis bacteriana (VB) sintomática) y de la ITU en todas las embarazadas. El diagnóstico de VB sintomática se hizo mediante la tinción de Gram del flujo vaginal según criterios de Nugent, asociada con leucocitos polimorfonucleares sobre 10 por campo al examen al fresco $(400 x)(24,25)$. El diagnóstico de vaginitis aeróbica se hizo con cultivo positivo para patógenos aeróbicos facultativos (Escherichia coli, Enterococcus spp., Staphylococcus spp. y Streptococcus grupo $\mathrm{B})$, asociada con disminución de flora lactobacilar y aumento de leucocitos polimorfonucleares sobre 10 por campo al examen al fresco $(26,27)$. La ITU se diagnosticó mediante el urocultivo positivo. La VB se trató con clindamicina oral $300 \mathrm{mg}$ cada 8 horas $x 7$ días o metronidazol oral 500 mg cada 8 horas $x$ 7 días. La vaginitis aeróbica se trató con ampicilina oral $500 \mathrm{mg}$ cada 6 horas $\times 7$ días, asociado a tratamiento local con clindamicina 1 óvulo diario por 1 semana. La ITU se trató con cefradina oral $500 \mathrm{mg}$ cada 6 horas x 7-10 días (E.coli) y con ampicilina oral 500 mg cada 6 horas x 7-10 días (cocos Gram positivo) (27).

Estudio histológico placentario. De los hallazgos sugerentes de infección ascendente aguda: corioamnionitis (presencia de leucocitos polimorfonucleares que infiltran amnios y corion con o sin necrosis) y funisitis (inflamación aguda del cordón umbilical con infiltración de leucocitos polimorfonucleares en la pared de los vasos umbilicales o gelatina de Warthon) (2,17-19).

Mortalidad perinatal y morbilidad neonatal precoz.
La etiología de la muerte fetal se estableció luego de analizar en conjunto los antecedentes maternos clínicos y de laboratorio con los hallazgos anatomopatológicos feto-placentarios. La causa de muerte neonatal dentro de los primeros 7 días de vida, se obtuvo mediante el análisis de los datos clínicos, de laboratorio y de la autopsia del neonato.

Las siguientes morbilidades severas se analizaron durante la primera semana de vida (28):

a. Sepsis neonatal comprobada: se diagnosticó en presencia de cultivo positivo de sangre, orina o líquido cefaloraquídeo.

b. Neumonía: se diagnosticó con cuadro clínico y radiológico compatible con o sin cultivo positivo de aspirado traqueal o de sangre.

c. Síndrome de dificultad respiratoria (SDR): se definió como el cuadro respiratorio con quejido y retracción torácica durante la fase inspiratoria, con requerimientos de $\mathrm{O} 2$ aumentado ( $\mathrm{FiO} 2>0,21$ ), con radiología compatible, gases arteriales alterados y ausencia de otras causas de enfermedades respiratorias.

d. Enterocolitis necrotizante: se diagnosticó en presencia de distensión abdominal, intolerancia alimentaria 24 horas o más, deposiciones sanguinolentas, con clara evidencia radiológica de aire intramural, de perforación, síndrome meconial o hallazgos específicos en la cirugía o autopsia.

e. Hemorragia intraventricular: se diagnosticó por el examen ultrasonográfico de la cabeza del neonato y se consideraron las hemorragias intraventriculares severas, grados III y IV.

f. Asfixia severa: se consideró cuando el neonato presentó test de Apgar de 3 puntos o menos a los 5 minutos de vida.

Se utilizó el resultado perinatal adverso compuesto, determinado por mortalidad y la morbilidad severa mencionada anteriormente (28).

Expresión de resultados. El principal resultado fue la frecuencia del nacimiento prematuro $<34$ semanas. Además se analizó la frecuencia, del parto prematuro <37 semanas y del resultado perinatal adverso compuesto (morbilidad/mortalidad) (28). Se midió la frecuencia de corioamnionitis histológica. Análisis estadístico de los datos. El tamaño de la muestra se calculó usando niveles de alfa de 0,05 y de beta de 0,20. Considerando una incidencia de trabajo de parto prematuro para los controles de $30 \%$ y de $4 \%$ para los casos, se estableció en 25 el número de sujetos necesarios por grupo (29). Se utilizó test de chi cuadrado y Odds Ratio (OR) con intervalo de confianza de $95 \%$ (IC95\%) en la comparación de proporciones. Se usó test T en la comparación de variables continuas. Se consideró significativo un valor de $p<0,05$. 


\section{RESULTADOS}

Características de la población. En el período en estudio se atendieron 73 embarazadas con embarazos únicos, sin partos de término y con nacimientos prematuros espontáneos previos. Se excluyeron 22 pacientes, 17 en que no se precisó el factor asociado al aborto y/o parto prematuro previo, por carencia de histopatología del feto y/o placenta, 1 con daño anatómico del cuello y 4 del grupo control que requirieron cerclaje, 2 por acortamiento cervi$\mathrm{cal}<25 \mathrm{~mm}$ y 2 por emergencia. Cincuenta y un pacientes cumplieron con los requisitos de admisión al estudio. Veintitrés embarazadas tuvieron cerclaje cervical (grupo estudio) y veintiocho se manejaron con conducta expectante sin cerclaje (grupo control). El cerclaje se colocó entre las 12 y 19 semanas (promedio 14,4 semanas). No hubo rechazos al ofrecimiento de cerclaje.

La historia obstétrica de las pacientes con cerclaje fue: 46 nacimientos prematuros únicos en total, promedio 2 por paciente [29 partos prematuros (entre 22 y 35 semanas), 17 abortos (entre 17 y 21 semanas)], mortalidad perinatal 78,3\% [(36/46) 23 muertes fetales y 13 muertes neonatales]. Los siguientes hallazgos clínicos y anátomopatológicos sugerentes de IBA presentaron estas pacientes: corioamnionitis histológica aguda 95,7\% (44/46), funisitis aguda $52,2 \%$ (24/46), neumonía congénita $26,1 \%$ [(12/46) todas con SISA, tres por SGB], RPM $21,7 \%$ (10/46), membranas prolapsadas $15,2 \%$ (7/46), DPPNI 10,9\% (5/46), ITU 8,7\% (4/46), ICV por SGB 4,4\% (2/46), corioamnionitis clínica 4,4\% (2/46) y sepsis neonatal 2,2\% (1/46).

La historia obstétrica de las pacientes sin cerclaje fue: 35 nacimientos prematuros únicos en total, promedio 1,3 por paciente, [29 partos prematuros (entre 22 y 35 semanas), 6 abortos (entre 17 y 21,5 semanas)], mortalidad perinatal 62,9\% [(22/35) 14 muertes fetales y 8 muertes neonatales]. Los siguientes hallazgos clínicos y anatomopatológicos sugerentes de IBA presentaron estas pacientes: corioamnionitis histológica aguda 97,1\% (34/35), funisitis aguda 57,1\% (20/35), neumonía congénita $31,4 \%$ [(11/35) todas con SISA], RPM 22,9\% (8/35), DPPNI 14,3\% (5/35), ITU 10,0\% (4/35), sepsis neonatal $8,6 \%(3 / 35)$ y un caso de corioamnionitis clínica, membranas prolapsadas, enterocolitis necrotizante y leucomalacia periventricular.

En la Tabla I se describen las características generales de la población. No hubo diferencias en edad materna, edad gestacional al inicio del control del embarazo, longitud cervical medida antes de las 20 y entre las 20-34 semanas y vía del parto entre ambos grupos. La edad gestacional al ingreso del estudio y el intervalo ingreso estudio-parto fueron significativamente diferentes entre los dos grupos.

La ITU se presentó en el 21,7\% (5/23) en el grupo estudio y en el $35,7 \%$ (10/28) en el grupo control. La infección cérvicovaginal ocurrió en el 73,9\% $(17 / 23)$ en el grupo estudio y $60,7 \%(17 / 28)$ en el grupo control. La edad gestacional de aparición y la mejoría microbiológica postratamiento de la infección genitourinaria se presentaron sin diferencias significativas en ambos grupos.

En la Tabla II se describen resultados del embarazo y del recién nacido. Los embarazos de término fueron significativamente más frecuentes en el grupo con cerclaje $22(95,7 \%)$ vs $18(64,3 \%) \sin$ cerclaje [OR: 12,2; IC95\% (1,43-104)]. La corioamnionitis histológica se redujo significativamente en el grupo estudio $4,3 \%(1 / 23)$ vs $32,1 \%(9 / 28)$ [OR 0,01; IC95\% (0,01-0,83)].

Las pacientes con cerclaje no presentaron complicaciones derivadas de su colocación ni de su uso (RPM, corioamnionitis clínica, infección puerperal).

En la Tabla III se describen los nacimientos prematuros espontáneos (abortos-partos) y el resultado perinatal. Las pacientes del grupo estudio tuvieron significativamente menos nacimientos espontáneos $<37$ semanas que las del grupo control, $4,3 \%(1 / 23)$ vs $35,7 \%(10 / 28)$ [OR 0,08 ; IC95\% $(0,09-0,70)]$ y $<34$ semanas, $4,3 \%(1 / 23)$ vs $28,6 \%$ (8/28) [OR 0,11; IC95\% (0,01-0,99).

El resultado perinatal adverso compuesto no presentó diferencias. Nacimientos prematuros. Grupo estudio: 1 parto de $31 \mathrm{~s}$ (1.544 g). Grupo

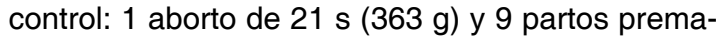
turos: $23 \mathrm{~s}(350 \mathrm{~g}), 23,6 \mathrm{~s}(630 \mathrm{~g}), 25 \mathrm{~s}(771 \mathrm{~g}), 27,3$ $\mathrm{s}(1.034 \mathrm{~g}), 27,5 \mathrm{~s}(1.289 \mathrm{~g}), 31,4 \mathrm{~s}(1.768 \mathrm{~g}), 33,2 \mathrm{~s}$ $(2.240 \mathrm{~g}), 34 \mathrm{~s}(2.110 \mathrm{~g})$ y $36,2 \mathrm{~s}(2.940 \mathrm{~g})$.

Resultado perinatal adverso compuesto (sólo en grupo control). Muertes: 3 muertes fetales por neumonía congénita, 1 neonatal precoz por neumonía congénita (muerte a las $2 \mathrm{~h}$ ). Morbilidad: 2 recién nacidos con neumonía y SDR, 1 niño con neumonía congénita. La infección se presentó en los 7 niños con resultados adversos del grupo control.

\section{DISCUSIÓN}

Los resultados del presente estudio muestran que el cerclaje cervical profiláctico, reduce la frecuencia del nacimiento prematuro $<37,<34$ semanas y la corioamnionitis histológica en pacientes con embarazos únicos, sin partos de término, cérvix $>25 \mathrm{~mm}$ e historia de partos prematuros y/o abortos de $2^{\circ}$ trimestre espontáneos asociados con IBA. 
Tabla I

CARACTERÍSTICAS DE LA POBLACIÓN EN ESTUDIO

\begin{tabular}{|c|c|c|c|}
\hline Variable & $\begin{array}{c}\text { Con cerclaje } \\
n=23\end{array}$ & $\begin{array}{c}\text { Sin cerclaje } \\
n=28\end{array}$ & Valor $p$ \\
\hline \multicolumn{4}{|l|}{ Edad materna (años) } \\
\hline Media (EE) & $26,9(1,3)$ & $25,1(1,2)$ & \multirow[t]{2}{*}{ NS } \\
\hline Rango & $18-41$ & $16-37$ & \\
\hline \multicolumn{4}{|c|}{$\begin{array}{l}\text { Edad gestacional } \\
\text { embarazo }\end{array}$} \\
\hline Media (EE) & $9,3(0,3)$ & $9,9(0,4)$ & \multirow[t]{2}{*}{ NS } \\
\hline Rango & $6-13$ & $7-15$ & \\
\hline \multicolumn{4}{|l|}{ Ingreso al estudio } \\
\hline Media (EE) & $11,4(0,5)$ & $23,3(0,6)$ & \multirow[t]{2}{*}{$<0,0001$} \\
\hline Rango & 6-14 & $20-29$ & \\
\hline \multicolumn{4}{|l|}{ Longitud cervical (mm ) } \\
\hline Antes de las 20 semanas & & & \multirow{3}{*}{ NS } \\
\hline Media (EE) & $32,6(0,9)$ & $36,2(1,6)^{\star}$ & \\
\hline Rango & $26-45$ & $30-40$ & \\
\hline \multicolumn{4}{|l|}{ Entre las $20-34$ semanas } \\
\hline Media (EE) & $29,1(1,4)$ & $29,7(0,6)$ & \multirow[t]{2}{*}{ NS } \\
\hline Rango & $15-40$ & $25-35$ & \\
\hline \multicolumn{4}{|c|}{ Intervalo ingreso estudio-parto (s) } \\
\hline Media (EE) & 26,9 & 11,9 & \multirow[t]{2}{*}{$<0,0001$} \\
\hline Rango & $19-33$ & $1-19$ & \\
\hline \multicolumn{4}{|c|}{ Infección durante el embarazo } \\
\hline Urinaria & & & \multirow{3}{*}{ NS } \\
\hline$N^{\circ}$ pacientes con ITU & $5(21,7 \%)$ & $10(35,7 \%)$ & \\
\hline Promedio episodios & 0,2 & 0,4 & \\
\hline \multicolumn{4}{|l|}{ Cérvicovaginal } \\
\hline $\mathrm{N}^{\circ}$ pacientes con ICV & $17(73,9 \%)$ & $17(60,7 \%)$ & \multirow[t]{2}{*}{ NS } \\
\hline Promedio episodios & 1,3 & 0,9 & \\
\hline \multicolumn{4}{|l|}{ Vía del parto } \\
\hline Vaginal & $11(47,8 \%)$ & $19(67,9 \%)$ & \multirow[t]{2}{*}{ NS } \\
\hline Cesárea & $12(52,2 \%)$ & $9(32,1 \%)$ & \\
\hline
\end{tabular}

EE: Error estándar. ITU: infección del tracto urinario. ICV: infección cérvicovaginal. *5/28 pacientes con medición del cérvix. NS: no significativo.

Estos hallazgos no están en concordancia con la conclusión de la revisión sistemática de la Base de Datos Cochrane (30), ni con los resultados de algunos estudios clínicos (10-13) que encuentran que el cerclaje profiláctico realizado sólo por los antecedentes y el cerclaje terapéutico efectuado en cuellos cortos no reducen el aborto y el parto prematuro.

El trabajo de CIRCLE trial no demostró reducción del nacimiento prematuro cuando comparó el cerclaje indicado por antecedentes con el cerclaje indicado por acortamiento cervical $<20 \mathrm{~mm}$ (15). Sin embargo, revisiones actuales han demostrado que el cerclaje cervical es efectivo en la reducción del parto prematuro cuando se indica en embarazadas con fetos únicos, nacimientos prematuros previos y longitud cervical $<25 \mathrm{~mm}$ y especialmente $<15 \mathrm{~mm}$ $(16,31,32)$. Similares resultados fueron descritos por Shirodkar y MacDonald 50 años atrás, cuando recomendaron cerclaje en embarazadas con nacimientos prematuros previos y cambios cervicales al examen digital $(33,34)$.

La principal diferencia con nuestro estudio es que seleccionamos una población con pobre historia obstétrica asociada con IBA (corioamnionitis histológica, funisitis, muerte perinatal, debida a 
Tabla II

RESULTADOS DEL EMBARAZO Y RECIÉN NACIDO

\begin{tabular}{|c|c|c|c|}
\hline & $\begin{array}{c}\text { Con cerclaje } \\
\quad n=23\end{array}$ & $\begin{array}{c}\text { Sin cerclaje } \\
n=28\end{array}$ & OR $(95 \%$ IC) \\
\hline \multicolumn{4}{|c|}{ Edad gestacional al parto (s) } \\
\hline $21-29,6$ & 0 & $6(21,4 \%)$ & $0,0002(0-1,67)$ \\
\hline $30-36,6$ & $1(4,3 \%)$ & $4(14,3 \%)$ & $0,27(0,03-2,6)$ \\
\hline$\geq 37$ & $22(95,7)$ & $18(64,3 \%)$ & $12,2(1,43-104)$ \\
\hline \multicolumn{4}{|c|}{ Peso recién nacido (g) } \\
\hline$<750$ & 0 & $3(10,7 \%)$ & $0,18(0,009-3,91)$ \\
\hline $750-1499$ & 0 & $3(10,7 \%)$ & $0,18(0,009-3,91)$ \\
\hline $1500-2499$ & $2(8,7 \%)$ & $3(10,7 \%)$ & $0,79(0,12-5,21)$ \\
\hline $\begin{array}{l}\geq 2500 \\
\text { Corioamnionitis hi }\end{array}$ & $21(91,3 \%)$ & $19(67,9 \%)$ & $4,97(0,95-25)$ \\
\hline $\mathrm{n}(\%)$ & $1(4,3 \%)$ & $9(32,1 \%)$ & $0,01(0,01-0,83)$ \\
\hline
\end{tabular}

Tabla III

NACIMIENTOS PREMATUROS ESPONTÁNEOS (ABORTOS - PARTOS) Y RESULTADO PERINATAL

\begin{tabular}{lccc}
\hline & $\begin{array}{c}\text { Con cerclaje } \\
\mathrm{n}=23\end{array}$ & $\begin{array}{c}\text { Sin cerclaje } \\
\mathrm{n}=28\end{array}$ & OR (95\% IC) \\
\hline $\begin{array}{l}\text { Nacimientos espontáneos <37s } \\
\mathrm{n}(\%)\end{array}$ & $1(4,3 \%)$ & $10(35,7 \%)$ & $0,08(0,09-0,70)$ \\
$\begin{array}{l}\text { Nacimientos espontáneos <34s } \\
\mathrm{n}(\%)\end{array}$ & $1(4,3 \%)$ & $8(28,6 \%)$ & $0,11(0,01-0,99)$ \\
$\begin{array}{l}\text { Nacimientos espontáneos <30s } \\
\mathrm{n}(\%)\end{array}$ & 0 & $6(21,4 \%)$ & $0,0002(0-1,67)$ \\
$\begin{array}{l}\text { Resultado perinatal adverso compuesto } \\
\mathrm{n}(\%)\end{array}$ & 0 & $7(25 \%)$ & $0,067(0,04-1,2)$ \\
$\begin{array}{l}\text { Ingreso UCl } \\
\mathrm{n}(\%)\end{array}$ & $1(4,3 \%)$ & $4(14,3 \%)^{*}$ & $0,27(0,03-2,6)$ \\
\end{tabular}

*3 fetos $<800 \mathrm{~g}$ no ingresaron a UCl por muerte.

infección congénita) y cervix $>25 \mathrm{~mm}$. Esperar el acortamiento cervical para hacer cerclaje es inconveniente, porque el riesgo de IMCA y de inflamación intraamniótica aumenta con menor longitud cervical (5-7).

Un hallazgo importante de este trabajo es que el cerclaje redujo la corioamnionitis histológica en embarazadas con alta frecuencia de infección génitourinaria. Esto puede interpretarse, que en pacientes con riesgo de aborto/parto prematuro por infección, el cerclaje puede impedir la progresión de la infección desde la vagina a la cavidad corio- amniótica. El moco cervical representa una efectiva barrera con propiedad antibacteriana, que impide el ascenso de las infecciones vaginales asociadas con parto prematuro (35). Disminuiría su eficacia frente a daños anatómicos y acortamiento de la longitud del cérvix. Así, en embarazos con estos factores de riesgo el cerclaje mejora la probabilidad de resultado favorable por la conservación del moco, de su capacidad antibacteriana y de la función anatómica del cérvix. Postulamos que en embarazadas con pobre historia obstétrica asociada con IBA, el cerclaje debe ofrecerse a estas pacientes durante 
el primer o segundo trimestre antes de que ocurra acortamiento cervical y aumente el riesgo de infección intraamniótica.

Este estudio tiene limitaciones. La más importante fue la selección del grupo control. Estas embarazadas ingresaron al trabajo después de las 20 semanas (media: 23,3 semanas) porque se derivaron al hospital en ese momento. Se siguió en ellas el criterio de conducta expectante, con vigilancia de la longitud cervical. Este error pudo reducirse porque, se excluyeron las pacientes controles que acortaron el cérvix a $<25 \mathrm{~mm}$, y porque no hubo diferencias en ambos grupos en frecuencia, y curación microbiológica postratamiento de la infección genitourinaria. Más aún, este sesgo pudo intervenir sobre la prevalencia de otros fenómenos, pero no sobre la clara asociación entre cerclaje profiláctico, buenos resultados obtenidos y descenso de la corioamnionitis histológica. Otra limitación fue la exclusión de 17 casos en que no se pudo precisar el factor asociado al aborto y/o parto prematuro previo, por carencia de estudio histopatológico del feto y/o placenta. Sin embargo, la decisión de no hacer estudio anátomo-patológico fue al azar, por lo que este error no debió modificar los resultados.

\section{CONCLUSIÓN}

En pacientes con nacimientos prematuros previos asociados con IBA, embarazo único y longitud cervical $>25 \mathrm{~mm}$, el cerclaje profiláctico, reduce la frecuencia del nacimiento prematuro $<37$ y $<34$ semanas y de la corioamnionitis histológica.

\section{REFERENCIAS}

1. Goldenberg RL, Culhane JF, lams JD, Romero R. Epidemiology and causes of preterm birth. Lancet 2008;371:75-84.

2. Al-Adnani M, Sebire NJ. The role of perinatal pathological examination in subclinical infection in obstetrics. Best Pract Res Clin Obstet Gynaecol 2007;21:505-21.

3. Ovalle A, Kakarieka E, Correa A, Vial MT, Aspillaga C. Estudio anátomo-clínico de las causas de muerte fetal. Rev Chil Obstet Ginecol 2005;70:303-12.

4. Ovalle A, Kakarieka E, Rencoret G, Fuentes A, Del Río MJ, Morong $\mathrm{C}$, et al. Factores asociados con el parto prematuro entre 22 y 34 semanas en un Hospital Público de Santiago. Rev Med Chile 2012;140:19-29.

5. Palacio M, Cobo T, Bosch J, Filella X, Navarro-Sastre FA, Ribes A, et al. Cervical length and gestational age at admission as predictors of intra-amniotic inflammation in preterm labor with intact membranes. Ultrasound Obstet Gynecol 2009;34: 441-7.

6. Holst RM, Jacobsson B, Hagberg H, Wennerholm UB. Cervical length in women in preterm labor with intact membranes: relationship to intra-amniotic in- flammation/microbial invasion, cervical inflammation and preterm delivery. Ultrasound Obstet Gynecol 2006;28:768-4.

7. Vaisbuch E, Hassan SS, Mazaki-Tovi S, Nhan-Chang $\mathrm{CH}$, Kusanovic JP, Chaiworapongsa T, et al. Patients with an asymptomatic short cervix $(<15 \mathrm{~mm})$ have a high rate of subclinical intraamniotic inflammation: implications for patient counseling Am J Obstet Gynecol 2010;202:433.e1-8.

8. Mcparland P, Jones G, Taylor D. Preterm labour and prematurity. Curr Obstet Gynaecol 2004:14:309-19

9. lams JD, Romero R, Culhane JF, Goldenberg RL. Primary, secondary, and tertiary interventions to reduce the morbidity and mortality of preterm birth. Lancet 2008;371:164-75.

10. Rush RW, Isaacs S, Mcpherson K, Jones L, Chalmers I, Grant A. A randomized controlled trial of cervical cerclage in women at high risk of spontaneous preterm delivery. Br J Obstet Gynaecol 1984;91:724-30.

11. Rust OA, Atlas RO, Reed J, Van Gaalen J, Balducci J. Revisiting the short cervix detected by transvaginal ultrasound in the second trimester: why cerclage therapy may not help. Am J Obstet Gynecol 2001;185:1098105.

12. Berghella V, Odido AO, Tolosa JE. Cerclage for prevention of preterm birth in women with a short cervix found on transvaginal ultrasound examination: a randomizaed trial Am J Obstet Gynecol 2004;191:1311-17.

13. To MS, Alfirevic Z, Heath VC, Cicero S, Cacho AM, Williamson RP, et al. Cervical cerclage for precention of preterm delivery in women with short cervix: a randomised controlled trial. Lancet 2004;363:1849-53.

14. Final report of the medical research Council/Royal College of Obstetricians and Gynaecologists multicentre randomized trial of cervical cerclage. MRC/RCOG Working Party on Cervical Cerclage. Br J Obstet Gynaecol 1993;100:516-23.

15. Simcox R, Seed PT, Bennett P, Teoh TG, Poston L, Andrew $\mathrm{H}$, et al. A randomized controlled trial of cervical scanning vs history to determine cerclage in women at high risk of preterm birth (CIRCLE trial). Am J Obstet Gynecol 2009;200:623.e1-623.e1-6.

16. Berghella V, Rafael TJ, Szychowski JM, Rust OA, Owen J. Cerclage for short cervix on ultrasonography in women with singleton gestations and previous preterm birth. Obstet Gynecol 2011;117:663-71.

17. Ovalle A, Martínez MA, Kakarieka ME, Gómez R, Torres J, Fuentes A, et al. Histopatología de la placenta en la rotura prematura de membranas de pretérmino. Relación con la microbiología aislada y con los resultados materno-neonatales. Rev Med Chile 1998;126:930-42.

18. Ovalle A, Gómez R, Martínez MA, Kakarieka E, Fuentes $A$, Aspillaga $C$, et al. Invasión microbiana de la cavidad amniótica en la rotura de membranas de pretérmino. Resultados materno-neonatales y patología placentaria según microorganismo aislado. Rev Med Chile 2005;133:51-61.

19. Park CW, Moon KC, Park JS, Jun JK, Romero R, Yoon $\mathrm{BH}$. The involvement of human amnion in histologic chorioamnionitis is an indicator that a fetal and an intra-amniotic inflammatory response is more likely and 
severe: clinical implications. Placenta 2009;30:56-61.

20. Kidron D, Bernheim J, Aviram R. Placental findings contributing to fetal death, a study of 120 stillbirths. Placenta 2009; 30: 700-4.

21. Sheiner E, Mazor-Drey E, Levy A. Asymptomatic bacteriuria during pregnancy. $\mathrm{J}$ Matern Fetal Neonatal Med 2009;22:423-7.

22. Håkansson S, Källén K. Impact and risk factors for early-onset group Bstreptococcal morbidity: analysis of a national, population-based cohort in Sweden 1997-2001. Br J Obstet Gynaecol 2006;113:1452-58.

23. Gómez R, Romero R, Nien JK, Medina L, Carstens M, $\mathrm{Kim} \mathrm{YM}$, et al. Idiopathic vaginal bleeding during pregnancy as the only clinical manifestation of intrauterine infection. J Matern Fetal Neonatal Med 2005;18:31-7.

24. Martínez MA, Ovalle A, Gaete AM, Lillo E, De La Fuente F, Araneda F, et al. Comparación de los criterios de Nugent y Spiegel para el diagnóstico de vaginosis bacteriana y análisis de los resultados discordantes por el método de Ison y Hay. Rev Med Chile 2011;139: 66-71.

25. Donders GG. Definition and classification of abnormal vaginal flora. Best Pract Res Clin Obstet Gynaecol. 2007; 21: 355-73.

26. Donders GG, Van Calsteren K, Bellen G, Reybrouck $\mathrm{R}$, Van Den Bosch T, Riphagen I, et al. Predictive value for preterm birth of abnormal vaginal flora, bacterial vaginosis and aerobic vaginitis during the first trimester of pregnancy. Br J Obstet Gynaecol 2009; 116:1315-24

27. Ovalle A, Gómez R. Infecciones y embarazo En: González R, Gómez R, Castro S, (eds). Guía Perinatal
Minsal. 1a edición. Cámara Chilena del Libro, 2003. pp 293-311.

28. Ovalle A, Romero R, Gómez R, Martínez MA, Nien JK, Ferrand $\mathrm{P}$, et al. Antibiotics and perinatal outcome in patients with preterm labor: possible beneficial effects in patients with endocervical inflammation. J Matern Fetal Neonatal Med 2006;19:453-64.

29. Lee T, Carpenter MW, Heber WW, Silver HM. Preterm premature rupture of membranes: risks of recurrent complications in the next pregnancy among a population-based sample of gravid women. Am J Obstet Gynecol 2003;188:209-13.

30. Drakeley AJ, Roberts D, Alfirevic Z. Sutura cervical (cerclaje) para prevenir la pérdida de embarazo en mujeres. La Biblioteca Cochrane Plus, 2007.

31. Owen J, Hankins G, lams JD, Berghella V, Sheffield JS, Perez-Delboy A, et al. Multicenter randomized trial of cerclage for preterm birth prevention in high-risk women with shortened midtrimester cervical length. Am J Obstet Gynecol 2009;201:375.e1-8.

32. Berghella V, Keeler SM, To MS, Althuisius SM, Rust $\mathrm{OA}$. Effectiveness of cerclage according to severity of cervical length shortening: a meta-analysis. Ultrasound Obstet Gynecol 2010;35:468-73

33. Mcdonald IA. Suture of the cervix for inevitable miscarriage. J Obstet Gynaecol Br Emp 1957;64:346-50.

34. Shirodkar VN. A new method of operative treatment for habitual abortions in the second trimester of pregnancy. Antiseptic 1955; 52:299-300.

35. Becher N, Waldorf KA, Hein M, Uldbjerg N. The cervical mucus plug: Structured review of the literature. Acta Obstet Gynecol Scand 2009; 88:502-13. 